\title{
Land Use/Land Cover and Accessibility: Implications of the Correlations for Land Use and Transport Planning
}

\section{Zhongqi Wang $^{1}$ (D) $\cdot$ Qi Han ${ }^{1} \cdot$ Bauke de Vries ${ }^{1}$}

Received: 2 May 2018 / Accepted: 8 October 2018 /

Published online: 25 October 2018

(C) The Author(s) 2018

\begin{abstract}
Land use and transport shape our cities. The central place of integrated land use transport strategy in improving urban sustainability has been a widespread acceptance. The purpose of this research is to investigate the relationship between land use/land cover types and transport characteristics. Traffic zones, as the spatial analysis unit, are generated by partitioning parcels with single land use/land cover type. Driving accessibility, cycling accessibility and walking accessibility are proposed to quantify the transport characteristics of traffic zones. Taking Eindhoven, the Netherlands as study area, the three accessibility patterns differ but all show strong positive spatial autocorrelation. Clustering method is adopted to synthesize accessibility indicators and group traffic zones with similar transport characteristics into clusters. An contingency table analysis indicates that land use/land cover types are significantly associated with clusters, on which the validation and quantification of experience in the relationship of land use/land cover and transport are based: residential area and commercial area mainly concentrate in high accessible clusters (6 and 7); industrial area and open space tend to favor clusters (4 and 6) with adequate driving accessibility; the high percentage of natural area in medium accessible clusters (4 and 5), especially in less accessible clusters (1,2 and 3) corroborates the low demands of natural area on mobility service. Clustering map and accessibility patterns can identify the gaps in coverage of mobility service and in efficiency of land use/ land cover pattern. The applicability of these tools is demonstrated by two cases. Recommendations for urban planning are obtained from this study considering both land use and transport aspects.
\end{abstract}

Keywords Accessibility · Transport characteristics · Land use/land cover type · Clustering analysis

Zhongqi Wang

Z.Wang.1@tue.nl

1 Information Systems in the Built Environment, Department of Built Environment, Eindhoven University of Technology, PO Box 513, 5600 MB Eindhoven, The Netherlands 


\section{Introduction}

As issues (e.g. traffic congestion and environmental degradation) emerging from urbanization and urban sprawl have attracted considerable attention, there is a growing consensus that integrating land use and transport is a vital pathway to urban sustainable development (Bertolini et al. 2005; Kenworthy and Laube 1996; Lautso et al. 2004; Weiss et al. 2018). The physical components of urban spatial structure, or more specially land use, are connected by transport systems, which facilitate the functioning of cities by passengers and freight movements. The implementation of both urban functions and transport construction need substantial land requirement and consumption. Since urban land is a scarce resource and must be used efficiently (Lambin and Meyfroidt 2011), urban development, both in land use and transport, should be planned and managed rather than develop spontaneously (Tian et al. 2017). The integration of land use and transport thereby is necessitated in urban planning and management. The definition and representation of land use systems and transport systems vary in research purpose and scale. Land use/land cover pattern can reflect the spatial characteristics of land use practices and location-specific transport capacity can be measured by accessibility, which refers to the ease with which anyplace of a certain area can be reached by individuals at a particular location using the mobility service of specific transport systems. The relationship between land use/land cover types and accessibility can provide new insights into the synergy of land use and transport planning at regional scale.

The relationship between land use and transport is complex and dynamic with various interactive effects (Litman and Steele 2017), which results in the breadth of land use transport literature. There is a long tradition in research of integrated land use and transport modelling and land use transport interaction (LUTI) model has come to significant fruition (Acheampong and Silva 2015). However, on account of a series of challenges such as model transparency, operability, parameter validity, computational performance, LUTI model has not progressed in practice as far as it is expected (Waddell 2011). With the enhancement of quality and availability in land use data, a trend towards empirical studies on this relationship has arisen. These researches can generally be divided into: pedestrian bike infrastructure and land use (Cervero and Duncan 2003; Duncan et al. 2010; Lee and Moudon 2006; Rodríguez et al. 2009; Wang et al. 2015; Witten et al. 2011); motorized infrastructure and land use (Duranton and Turner 2012; Giuliano et al. 2012; Horner and Schleith 2012; Stanilov 2003; Vandenbulcke et al. 2009). Rodríguez et al. (2009) pointed out that higher levels of walking accessibility can intensify population density, availability of retail and land use mix. Stanilov (2003) proposed integral accessibility, which assesses the ease of access to high-class roads, to explore the impact of transport networks on land use pattern. The result suggests that the priorities of accessible locations allocation are: commercial, industrial, multi-family, medium-density single-family and low-density single-family uses. In addition to the above mentioned two categories, urban rail system (tram and metro) is taken into account in studies at metropolitan scale (King 2011; Ratner and Goetz 2013). Ratner and Goetz (2013) found that the extended light rail transit increased residential density in station areas of Denver. All of these attempts confirm the fact that transport is inextricably related to land use and development. However, the transport impacts vary in mobility service provided by transport infrastructure. Little 
attention has been paid to the integrated effects of pedestrian bike infrastructure and motorized infrastructure. Moreover, besides land use/land cover change driven by transport networks (Chaudhuri and Clarke 2015; Mothorpe et al. 2013; Müller et al. 2010; Patarasuk 2013), there is a lack of investigation into the transport characteristics of different land use/land cover types, which can bring the perspective of integrated land use transport to urban spatial planning.

The objectives of this study therefore are: (1) to quantify transport characteristics of an area; (2) to investigate the relationship between land use/land cover types and transport characteristics; (3) to discuss the potential use of the measures of transport characteristics and the aforementioned relationship in urban planning and management. A series of measures are developed as driving accessibility, cycling accessibility and walking accessibility to capture the mobility service of pedestrian infrastructure, bike infrastructure and motorized infrastructure, respectively. We adopted hierarchical clustering to group spatial units in terms of transport characteristics which are quantified by accessibility, and then investigated the relationship between land use/land cover types and accessibility. The conclusions about this relationship are drawn from the contingency table analysis of land use/land cover types and transport clusters with Chi-square test. On the basis of the relationship, combined with accessibility measurements and clustering method (agglomerative hierarchical clustering using Ward's method), this study provides an operational and applicable approach, which can identify the gaps in coverage of mobility service and in efficiency of land use/land cover pattern. Two cases are designed to exhibit the effectiveness and applicability of the proposed approach.

With discussions on related literatures, this section has introduced the purpose and scope of this paper. "Study Area and Data" section goes into the study area and data sources. Methods are introduced in "Methods" section. "Results" section presents the results and illustrates how the results can be applied in planning practice. Conclusions and policy recommendations are followed in "Discussion and Conclusions" section.

\section{Study Area and Data}

Eindhoven is the fifth largest city in the Netherlands with a total area of $88.84 \mathrm{~km}^{2}$ and a population of 225,000 (November 2015). It is known as a major technological and industrial hub lying in the southern part of the country, between the economic centers of the Dutch Randstad conurbation, the Ruhr conurbation of Central Germany, Brussels and Antwerp. Industries are mainly located in the outskirts of the city. Influenced by the concept of neighborhood (Yang and Timmermans 2014), urban center and surrounding areas are distributed alternatively with residential and commercial uses. Eindhoven has relatively complete transport systems, covering foot, bike, car, bus, train (two stations support long-distance public transport) and plane (Eindhoven airport is the second largest one in the Netherlands). The road networks comprise motorways, arterials, collectors and local roads. The ring road A2 Eindhoven, with one uninterrupted main track and two parallel roads, connects the local road networks to the national network, which supports express traffic passing the city region as well as trips between Eindhoven and neighboring towns.

For analyzing the relationship between transport characteristics and land use/land cover types, the data used in this study is composed of two parts: transport network data 
obtained from Open Street Map (OSM) and land use/land cover data obtained from Dutch land registry (NL: Kadaster). The OSM data of 2013 contains Eindhoven road networks and surrounding connected roads (see Fig. 1), which are line geometries characterized by length and max allowed speed. These can be used to construct a routable topology graph (directed, weighted and connected), which consists of nodes and edges, so that any well-known route search algorithm can be applied (Naumann and Kovalyov 2017). For Eindhoven road networks (surrounding connected roads are not included), the car network contains 4755 nodes and 6957 links; the bike network contains 4646 nodes and 6781 links; the foot network contains 4654 nodes and 6819 links (Zhang et al. 2011). The total length of transport networks is $3707 \mathrm{~km}$, which encompasses approximately $100 \mathrm{~km}$ highways (TomTom 2016).

The spatial land use database of Statistics Netherlands (NL: BBG, Bestand Bodemgebruik), BBG2012 (CBS 2012), contains digital geometry of the boundaries of land use/land cover in Eindhoven in 2012 (see Fig. 1). The entities of BBG2012 are parcels with single land use/land cover type in vector format. Compared to raster format, each parcel, which is enclosed by boundaries with various length, can vary in shape and size. In addition to land use/land cover type, attributes like shape, border length and area are stored for each parcel. The 970 parcels of Eindhoven belong in 7 land use/land cover types, including transport area, built area, semi-built area, recreation area, agricultural area, forest and open natural area, inland water, each of which is subdivided into a number of categories. In this study, these original land use/land cover types in BBG2012 are reclassified as: transport, residential, commercial, industrial, natural area and open

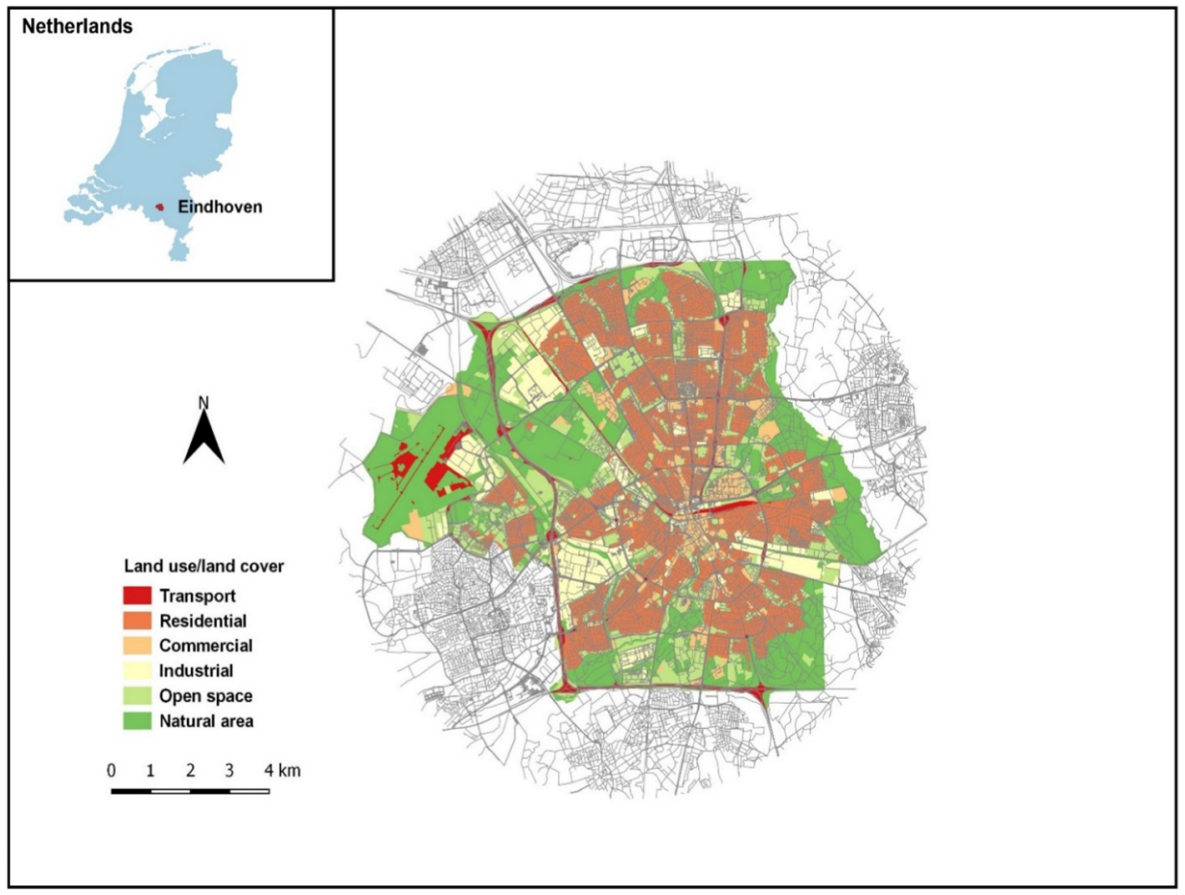

Fig. 1 The land use/land cover pattern of Eindhoven and transport network 
space. The proportions of these 6 new defined types of Eindhoven are $10.9 \%$, $31.4 \%, 6.2 \%, 9.6 \%, 15.9 \%$ and $26.1 \%$, respectively.

\section{Methods}

\section{Spatial Unit and Construction of OD Matrix}

The spatial frame of this study necessitates the use of zone-based accessibility measures. The commonly used spatial units are administrative division and grid cell. As study area ranges from a city to a country, even the whole world, the type, shape and size of spatial unit differ in research purposes and there is no consensus over them. Administrative divisions are often taken as traffic analysis zones in transport related studies with large study areas, while grid cells are principally employed in land use/land cover related researches at a finer level. To create accessibility patterns and further investigate the relationship between land use/land cover types and accessibility, the spatial unit of analysis used in this study factors in the following considerations: modifiable areal unit problem (MAUP), the homogeneity of transport characteristics and the uniqueness of land use/land cover type.

First of all, the partitioning scheme of study area can affect the calculation results of accessibility, which is known as MAUP (Kwan and Weber 2008). In this study, if each spatial unit is represented by a point, MAUP refers to the errors in travel time measured between locations due to the size and shape of spatial unit. The consistence of size and shape and resulting uniform spatial distribution of grid cells can handle MAUP efficiently. Secondly, the variance in transport characteristics would appear in spatial units with excessive size. Small size can ensure the homogeneity of transport characteristics but smaller unit is necessarily accompanied by a 'side effect' - increasing computation burden. With the improvement of computing power, more and more high grid cell resolutions have been applied, some of which are $100 \times 100 \mathrm{~m}$ (Cao et al. 2012), $200 \times 200 \mathrm{~m}$ (Pei et al. 2014) and $250 \times 250 \mathrm{~m}$ (Chaudhuri and Clarke 2015). Thirdly and lastly, one grid cell may cover several different land use/land cover types. The complicated representation of land use/land cover type is an obstacle to establish linkages with accessibility, which requires the uniqueness of land use/land cover type in spatial units. To strike a balance between these three considerations, in this study, parcels with single land use/land cover type in BBG2012 were taken as the basis for analysis unit division, and in QGIS 2.18, polygon divider plugin was used to divide parcels into smaller 'squarish' sections with approximately same size by the implementation of Brent's method (Brent 1971). No matter in reality or in BBG2012 dataset, parcels are commonly irregular shaped. So, most of the output spatial units can be in squarish shape with predefined size, while the edge of a parcel is always split into irregular pieces. The output spatial units inherit attributes including land use/land cover type from their parent and serve as traffic zones for measuring accessibility. In terms of land use/land cover types, traffic zones, the spatial units of this study, are divided into: residential traffic zone (RTZ), commercial traffic zone (CTZ), industrial traffic zone (ITZ), open space traffic zone (OSTZ), natural area traffic zone (NATZ) and transport traffic zone (TTZ). 
In the Netherlands, the houses are geo-coded at 4-digit and 6-digit postcode level. For a 6-digit postcode area, it consists of at most about 20 houses (Debrezion et al. 2011), which results in a phenomenon that 6-digit areas near city center are smaller than the outskirts where one postcode represents a larger area. Nevertheless, the 6-digit postcode division is an appropriate scale for reference because it takes into account population distribution. Eindhoven has 5218 6-digit postcode districts and the average area is $17,025 \mathrm{~m}^{2}$. Roughly in line with 6-digit postcode divisions, the size of traffic zone was set as $16,900 \mathrm{~m}^{2}(130 \mathrm{~m} \times 130 \mathrm{~m})$ and the study area hereby was partitioned into 5355 traffic zones.

For accessibility measures, these 5355 traffic zones were converted into their centroids which correspond to the geometric centers. These centroids are origins as well as destinations. The total number of origin-destination (OD) pairs is 28,670,670. The road graph was built in QGIS 2.18 through Python Console and each origin (destination) point was tied to the road graph. The paths with minimum travel time from origin $i$ to all the other destinations were searched by Dijkstra's algorithm.

\section{Accessibility Measurement}

In this study, transport infrastructure means road networks and accessibility, which captures the mobility service provided by road networks, is proposed to quantify the transport characteristics of a traffic zone. The main modes of inner-city transport in Eindhoven are driving, cycling and walking. Bus-based public transit is just an extension of motorized service, which utilizes infrastructure chiefly designed and constructed for driving. Therefore, transport characteristics of a traffic zone are depicted from three aspects: driving accessibility, cycling accessibility and walking accessibility. It is assumed that any location of developed cities (e.g. Eindhoven) is accessible. The intuitive approach to evaluate accessibility would be travel time. The accessibility measures of a traffic zone hereby are based on average travel time from that to all the other accessible traffic zones. Furthermore, a negative exponential function, as the most widely used and appropriate form associated with travel behavior theory (Handy and Niemeier 1997), is applied to estimate the impedance function in accessibility. Accessibility indicators: driving accessibility $A^{D}$, cycling accessibility $A^{C}{ }_{i}$ and walking accessibility $A^{W}{ }_{i}$ have the following equations:

$$
\begin{aligned}
& A_{i}^{D}=e^{-f^{D}(i)} \\
& A_{i}^{C}=e^{-f^{C}(i)} \\
& A_{i}^{W}=e^{-f^{W}(i)}
\end{aligned}
$$

where $f^{D}(i), f^{C}(i)$ and $f^{W}(i)$ are the travel time functions for driving, cycling and walking, respectively. 
Among the three modes, the driving accessibility needs to consider the effects of traffic congestion, otherwise, the driving time is underestimated during peak hours. Considering the obvious traffic performance differences in peak and off-peak hours, the driving time function has two components: average driving time during off-peak hours, and average driving time during peak hours, which describes reduced accessibility under rush hour condition. It can be expressed in the following manner:

$$
f^{D}(i)=\beta_{\text {peak }} \cdot \sum_{j \in Z_{i}^{D}} t_{i j}^{D}\left(1+\alpha_{k}\right) / N_{i}^{D}+\beta_{o f f-p e a k} \cdot \sum_{j \in Z_{i}^{D}} t_{i j}^{D} / N_{i}^{D}
$$

where, $t^{D_{i j}}$ is the minimum driving time from origin $i$ to destination $j, \alpha_{k}$ is the congestion parameter for roads in type $k, Z^{D}{ }_{i}$ is the driving trip area of origin $i$, $N^{D_{i}}$ is the number of destinations in $Z^{D_{i}}, \beta_{\text {peak }}$ and $\beta_{\text {off-peak }}$ are coefficients to measure the contribution of peak hours condition and off-peak hours condition to the whole day traffic performance. $Z^{D_{i}}$ defines the movement scope of driving from origin $i$. Likewise, there are $Z^{C}{ }_{i}$ for cycling and $Z^{W}{ }_{i}$ for walking. The scope is determined by the movement capacity of different travel modes. For driving, the trip area $Z^{D_{i}}$ is the whole city and thus, $N^{D_{i}}$ is the total number of destinations in Eindhoven. The value of $\alpha_{k}$ is determined by road type. According to the traffic congestion statistics of Eindhoven, during peak hours, the travel time increases $11 \%$ in highways and $25 \%$ in non-highways (TomTom 2016). Though non-highways actually contain several subtypes, the validation of $\alpha$ is limited by reliable empirical data. Therefore, given $k=1$ denotes highways and $k=2$ denotes non-highways, $\alpha_{1}=0.11$ and $\alpha_{2}=0.25$. Equal weighting is applied to the values of $\beta$, i.e. $\beta_{\text {peak }}=0.5$ and $\beta_{\text {off-peak }}=0.5$, which could be further validated by experts if needed.

The trip area setting of cycling and walking refers to Dur and Yigitcanlar (2015). For cycling, the trip area of origin $i$ is a circular region of $3 \mathrm{~km}$ radius with center $i$. An example of a cycling trip area is shown in Fig. 2. For each origin, only traffic zones within $3 \mathrm{~km}$ (the outer circle in Fig. 2) are used as destinations to calculate the cycling accessibility. The cycling time function is given by:

$$
f^{C}(i)=\sum_{j \in Z_{i}^{C}} t_{i j}^{C} / N_{i}^{C}
$$

where $t^{C}{ }_{i j}$ is the minimum cycling time from origin $i$ to destination $j, Z^{C}$ is the cycling trip area of origin $i, N^{C}$ is the number of destinations in $Z^{C}$. Walking accessibility is measured in a similar approach with cycling accessibility, except the trip area of origin $i$ is a circular region of $800 \mathrm{~m}$ radius with center $i$. For each origin, only traffic zones within $800 \mathrm{~m}$ (the inner circle in Fig. 2) are used as destinations to calculate the walking accessibility. So walking time function has the following equation:

$$
f^{W}(i)=\sum_{j \in Z_{i}^{W}} t_{i j}^{W} / N_{i}^{W}
$$

where $t^{W_{i j}}$ is the minimum walking time from origin $i$ to destination $j, Z^{W_{i}}$ is the walking trip area of origin $i, N^{W}{ }_{i}$ is the number of destinations in $Z^{W}$. The cycling speed was set 
as $15 \mathrm{~km} / \mathrm{h}$ and walking speed was set as $5 \mathrm{~km} / \mathrm{h}$. Road speed limits were used to set the driving speed.

\section{Spatial Pattern and Cluster Analysis}

For visualizing the accessibility disparity, the traffic zones were divided into 6 classes by driving, cycling and walking accessibility, respectively. The classification is based on Jenks natural breaks, which is a method to place data values into discrete categories with minimum squared deviation within classes and maximum squared deviation between classes. Moran's $I$ was calculated to measure the spatial autocorrelation of accessibility. Moran's I, which was initially proposed by Moran (1948) and popularized by Cliff and Ord (1973), can help understand the degree to which one object is similar to other nearby objects. The spatial pattern can be simply characterized as dispersion, randomness and clustering. In general, the value of Moran's $I$ varies on a scale between -1 and 1 . Positive values indicate positive spatial autocorrelation (clustering); negative values indicate negative spatial autocorrelation (dispersed); a randomly dispersed pattern results in a value close to 0.

Agglomerative hierarchical clustering using Ward's method was employed to synthesize accessibility indicators so that the transport characteristics of each traffic zone can be comprehensively estimated. Each resulting cluster represents specific transport characteristics which are identified by accessibility values. Hierarchical clustering, including agglomerative and divisive two types, is a commonly used unsupervised statistical method for clustering data. Agglomerative hierarchical clustering starts with each object in its own single-element cluster, and then at each step, pairs of clusters are merged in order of decreasing similarity, which is measured by distance between

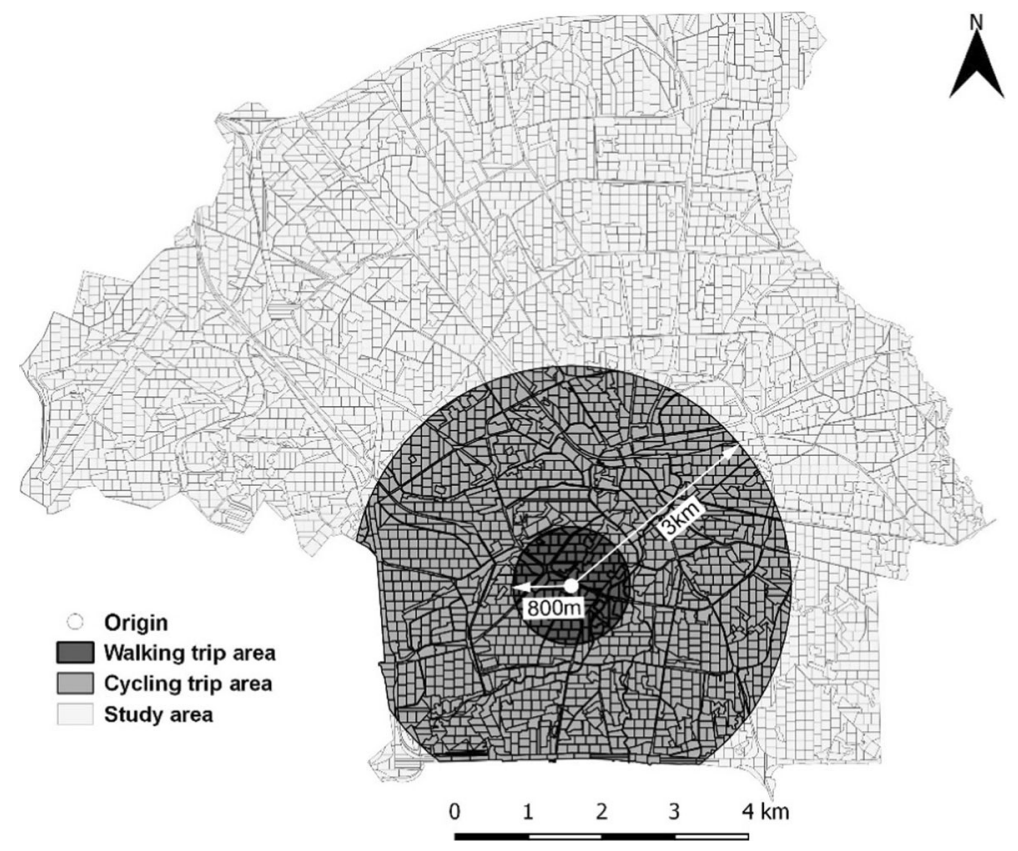

Fig. 2 Examples of cycling and walking trip areas 
clusters. The Ward's method evaluates the distance between classes by variance approach and it attempts to minimize the error sum of squares (ESS) of any possible cluster pair at each step of grouping (Murtagh and Legendre 2014).

\section{Results}

\section{Spatial Accessibility Pattern}

We visualized and mapped the calculations of driving accessibility (Fig. 3a), cycling accessibility (Fig. 3b) and walking accessibility (Fig. 3c). The three accessibility patterns show how mobility service for driving, cycling and walking varies spatially with the current transport infrastructure. In Eindhoven, driving accessibility decreases from urban center to remote areas (see Fig. 3a). The disparity of driving convenience disclosed by driving accessibility pattern is mainly caused by the motorized network, though only considering inner-city transport increases the inaccessibility of urban edge. The least accessible areas (see yellow traffic zones in Fig. 3a) for driving are concentrated in A2. A2 is controlled-access highway which is designed for high-speed cross-region vehicular traffic. However, in spite of the speed limit of A2 is absolutely high, A2 is less driving accessible for inner-city transport due to its limited entrances and exits. Compared with
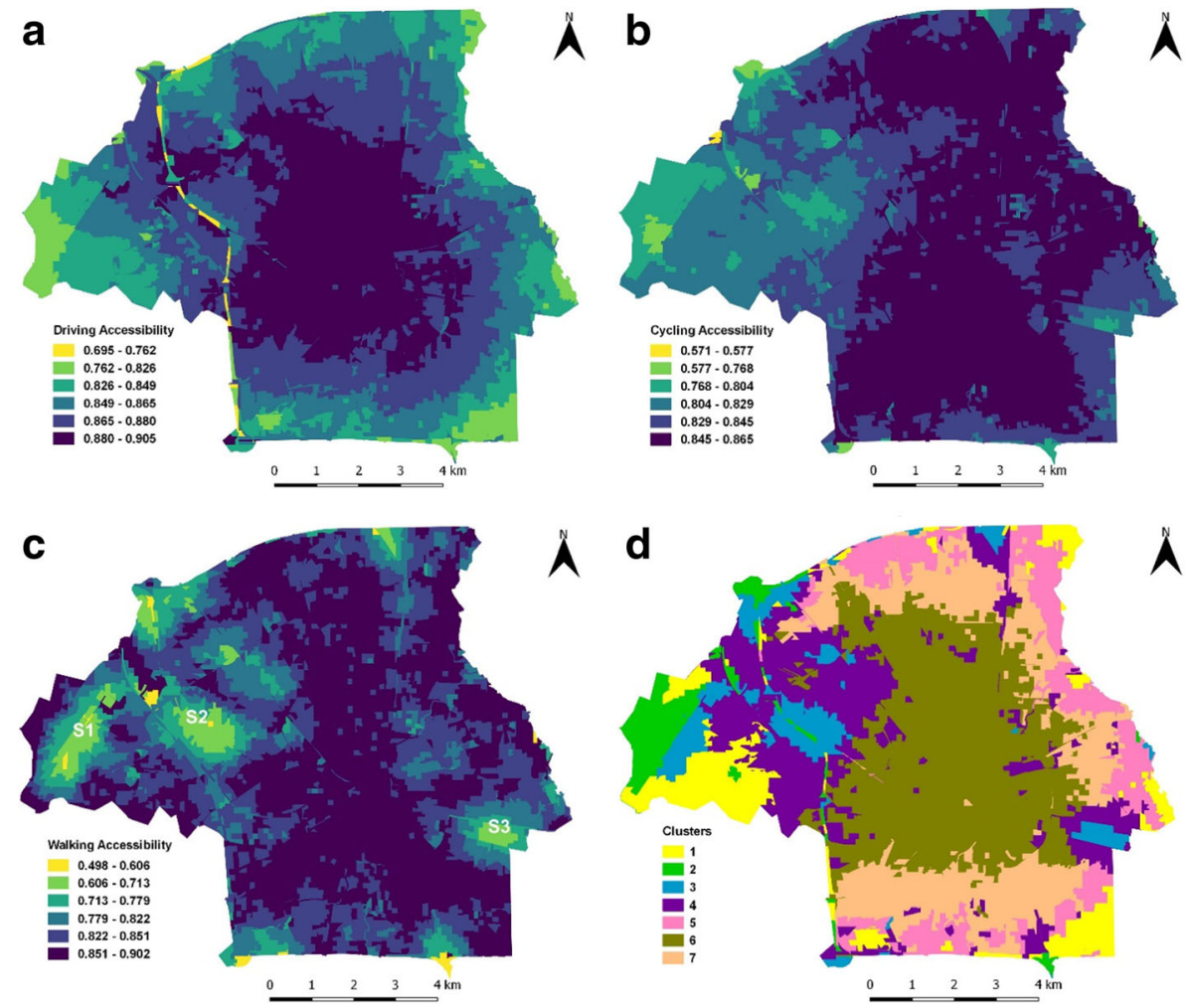

Fig. 3 Accessibility patterns and clustering map: a driving accessibility pattern, $\mathbf{b}$ cycling accessibility pattern, c walking accessibility pattern and $\mathbf{d}$ subdivisions of the study area in 7 clusters 
driving accessibility whose trip area is the whole city, the accessibility of medium-distance cycling is vulnerable to areas with sparse roads, such as natural area and open space. As a consequence, the cycling infrastructure provides citizens with more favorable cycling condition in the middle and east of the study area than western Eindhoven where large tracts of natural area and open space are located (see Fig. 3b). For walking, traffic zones with high walking accessibility shows a north-south distribution (see Fig. 3c), which corresponds to the distribution of residential and commercial areas (see Fig. 1).

A comparison of Fig. 3a-c locates three particular less accessible areas for driving, cycling and walking, which are labelled as S1, S2 and S3 in Fig. 3c. S1 overlaps Eindhoven airport and most of S2 falls into a piece of natural area. S3 situates along the banks of Eindhovens Kanaal (a canal). The depression of accessibility in S1 results from the closed nature around the airport. The relatively sparse roads, which accommodates the low travel generation and attraction in natural area, lead to the travel inconvenience in S2. In S3, the canal impedes the connection of local roads on both sides of it. In contrast with the numerous bridges over Dommel (another river across Eindhoven), there are only 3 bridges over Eindhovens Kanaal within $3.3 \mathrm{~km}$. Moreover, there is a fenced industrial park with a length of about $2 \mathrm{~km}$ and a width of about $0.5 \mathrm{~km}$ in S3. The enclosure of the industrial park is another reason for the low accessibility of S3. The industrial park only has two entrances \& exits, one is on the east side and the other one is on the west side, which damage the connectivity between inside roads of industrial park and urban road networks.

All these accessibility patterns show spatial agglomeration: to a certain extent, dark areas (high accessibility) and bright areas (low accessibility) are individually concentrated (Fig. 3a-c). The Moran's I results of driving, cycling and walking accessibility demonstrate strong positive spatial autocorrelation (Table 1). Z-scores which are achieved by Monte Carlo method indicate that the results are statistically significant $(p<0.001)$. In reality, the effects of transport infrastructure radiate outwards across surrounding areas, and as a result, neighboring places within the area of influence have similar transport characteristics. The calculated high positive Moran's I values validate this phenomenon. Additionally, it can be seen from Fig. 1 that there are several agglomerations of land use/land cover. The positive spatial autocorrelation of accessibility corresponds to the land use/land cover agglomeration, if any linkage between land use/land cover types and accessibility exists, which is discussed in "Cluster Analysis: A Synthesis of Accessibility".

\section{Cluster Analysis: A Synthesis of Accessibility}

We divided the traffic zones into clusters according to the accessibility differences of driving, cycling and walking. $F$-statistics (Table 2) and $t^{2}$-statistics, combined with

Table 1 Global spatial analysis

\begin{tabular}{llll}
\hline & Driving accessibility & Cycling accessibility & Walking accessibility \\
\hline Moran's $I$ & 0.771833 & 0.866856 & 0.842676 \\
Z-score & 77.1255 & 89.1865 & 87.5478 \\
\hline
\end{tabular}


Table 2 Descriptive statistics of cluster centers

\begin{tabular}{|c|c|c|c|c|c|c|c|c|}
\hline \multirow[t]{2}{*}{ Variable } & \multicolumn{8}{|c|}{ Cluster center } \\
\hline & 1 & 2 & 3 & 4 & 5 & 6 & 7 & $F$-statistics \\
\hline Driving accessibility a & -0.8932 & -1.6307 & 0.1395 & 0.7024 & -0.1799 & 1.2945 & 0.5674 & 2164.691 \\
\hline Average driving time ${ }^{b}$ & 11.0851 & 12.4630 & 9.2764 & 8.3126 & 9.8215 & 7.3141 & 8.5372 & \\
\hline Cycling accessibility a & -0.0760 & -1.7384 & -0.8900 & 0.1805 & 0.7406 & 0.8254 & 0.9579 & 2714.913 \\
\hline Average cycling time ${ }^{c}$ & 11.5971 & 14.9070 & 13.1850 & 11.1035 & 10.0465 & 9.8877 & 9.6422 & \\
\hline Walking accessibility a & 0.3107 & -0.5036 & -2.0213 & 0.0518 & 0.6778 & 0.7448 & 0.7398 & 1780.757 \\
\hline Average walking time ${ }^{d}$ & 11.2324 & 14.9654 & 22.0026 & 12.3106 & 9.6866 & 9.4084 & 9.4279 & \\
\hline
\end{tabular}

a The values have been normalized to zero mean and unit variance

$\mathrm{b}$ The mean of driving time calculated by Eq. (4)

${ }^{c}$ The mean of cycling time calculated by Eq. (5)

${ }^{d}$ The mean of walking time calculated by Eq. (6)

consideration of the number of land use/land cover types, suggest that seven clusters is the optimum scheme. The statistics of each cluster are compiled in Table 2. The mean accessibility values of each cluster are given as the cluster center to represent transport characteristics of affiliated traffic zones. Average travel time which corresponds to accessibility is listed on odd rows. The classification results are presented graphically in Fig. 3d, which can be explained as a superimposition of Fig. 3a-c in a new color scheme for clusters.

As illustrated in Table 2, among these seven clusters, the comprehensive accessibility of cluster 6 and cluster 7 are obviously better than the others. The driving accessibility and walking accessibility of cluster 6 are the highest and the cycling accessibility is only second to cluster 7 . The cycling accessibility of cluster 7 is highest, meanwhile, the walking accessibility and driving accessibility rank second and third out of seven clusters, respectively. On the other hand, compared with cluster 6 and cluster 7 , the poor performance of cluster 1, cluster 2 and cluster 3 is apparent. Traffic zones in cluster 2 are inconvenient for driving and cycling, which is indicated by the lowest driving accessibility and cycling accessibility of cluster 2 . Though the driving accessibility of cluster 3 is middling, the pedestrian and bike circumstance of traffic zones in cluster 3 is unfavorable, which is indicated by the lowest walking accessibility and second lowest cycling accessibility. Cluster 1 is also no better off except its medium walking accessibility. The comprehensive accessibility of cluster 4 and cluster 5 are mediocre but these two clusters have their own advantages. Cluster 4 is relatively prominent in driving (second highest driving accessibility) while cluster 5 performs well in cycling (third highest cycling accessibility) and walking (third highest walking accessibility).

To investigate the relationship between land use/land cover types and accessibility, the statistics for the distribution of seven clusters in each land use/land cover type and the distribution of land use/land cover types in seven clusters were made. The results are presented in a $6 \times 7$ contingency table (Table 3 ). The 
Table 3 The distributions of land use types and clusters

\begin{tabular}{|c|c|c|c|c|c|c|c|c|c|}
\hline & & \multicolumn{8}{|l|}{ Cluster } \\
\hline & & 1 & 2 & 3 & 4 & 5 & 6 & 7 & Total \\
\hline \multirow[t]{4}{*}{ RTZ } & Count & 7 & 0 & 0 & 143 & 103 & 852 & 576 & 1681 \\
\hline & $\%$ within land use & $0.4 \%$ & $0.0 \%$ & $0.0 \%$ & $8.5 \%$ & $6.1 \%$ & $50.7 \%$ & $34.3 \%$ & $100.0 \%$ \\
\hline & $\%$ within cluster & $1.4 \%$ & $0.0 \%$ & $0.0 \%$ & $14.5 \%$ & $16.9 \%$ & $50.9 \%$ & $55.8 \%$ & $31.4 \%$ \\
\hline & $\%$ of Total & $0.1 \%$ & $0.0 \%$ & $0.0 \%$ & $2.7 \%$ & $1.9 \%$ & $15.9 \%$ & $10.8 \%$ & $31.4 \%$ \\
\hline \multirow[t]{4}{*}{ CTZ } & Count & 14 & 2 & 2 & 53 & 41 & 173 & 48 & 333 \\
\hline & $\%$ within land use & $4.2 \%$ & $0.6 \%$ & $0.6 \%$ & $15.9 \%$ & $12.3 \%$ & $52.0 \%$ & $14.4 \%$ & $100 \%$ \\
\hline & $\%$ within cluster & $2.8 \%$ & $0.9 \%$ & $0.6 \%$ & $5.4 \%$ & $6.7 \%$ & $10.3 \%$ & $4.6 \%$ & $6.2 \%$ \\
\hline & $\%$ of Total & $0.3 \%$ & $0.0 \%$ & $0.0 \%$ & $1.0 \%$ & $0.8 \%$ & $3.2 \%$ & $0.9 \%$ & $6.2 \%$ \\
\hline \multirow[t]{4}{*}{ ITZ } & Count & 11 & 2 & 42 & 168 & 27 & 204 & 60 & 514 \\
\hline & $\%$ within land use & $2.1 \%$ & $0.4 \%$ & $8.2 \%$ & $32.7 \%$ & $5.3 \%$ & $39.7 \%$ & $11.7 \%$ & $100.0 \%$ \\
\hline & $\%$ within cluster & $2.2 \%$ & $0.9 \%$ & $12.6 \%$ & $17.0 \%$ & $4.4 \%$ & $12.2 \%$ & $5.8 \%$ & $9.6 \%$ \\
\hline & $\%$ of Total & $0.2 \%$ & $0.0 \%$ & $0.8 \%$ & $3.1 \%$ & $0.5 \%$ & $3.8 \%$ & $1.1 \%$ & $9.6 \%$ \\
\hline \multirow[t]{4}{*}{ OSTZ } & Count & 112 & 15 & 62 & 196 & 100 & 214 & 151 & 850 \\
\hline & $\%$ within land use & $13.2 \%$ & $1.8 \%$ & $7.3 \%$ & $23.1 \%$ & $11.8 \%$ & $25.2 \%$ & $17.8 \%$ & $100.0 \%$ \\
\hline & $\%$ within cluster & $22.7 \%$ & $6.7 \%$ & $18.6 \%$ & $19.8 \%$ & $16.4 \%$ & $12.8 \%$ & $14.6 \%$ & $15.9 \%$ \\
\hline & $\%$ of Total & $2.1 \%$ & $0.3 \%$ & $1.2 \%$ & $3.7 \%$ & $1.9 \%$ & $4.0 \%$ & $2.8 \%$ & $15.9 \%$ \\
\hline \multirow[t]{4}{*}{ NATZ } & Count & 293 & 121 & 165 & 314 & 302 & 77 & 123 & 1395 \\
\hline & $\%$ within land use & $21.0 \%$ & $8.7 \%$ & $11.8 \%$ & $22.5 \%$ & $21.6 \%$ & $5.5 \%$ & $8.8 \%$ & $100.0 \%$ \\
\hline & $\%$ within cluster & $59.4 \%$ & $54.3 \%$ & $49.5 \%$ & $31.8 \%$ & $49.5 \%$ & $4.6 \%$ & $11.9 \%$ & $26.1 \%$ \\
\hline & $\%$ of Total & $5.5 \%$ & $2.3 \%$ & $3.1 \%$ & $5.9 \%$ & $5.6 \%$ & $1.4 \%$ & $2.3 \%$ & $26.1 \%$ \\
\hline \multirow[t]{8}{*}{$\mathrm{TTZ}$} & Count & 56 & 83 & 62 & 114 & 37 & 155 & 75 & 582 \\
\hline & $\%$ within land use & $9.6 \%$ & $14.3 \%$ & $10.7 \%$ & $19.6 \%$ & $6.4 \%$ & $26.6 \%$ & $12.9 \%$ & $100.0 \%$ \\
\hline & $\%$ within cluster & $11.4 \%$ & $37.2 \%$ & $18.6 \%$ & $11.5 \%$ & $6.1 \%$ & $9.3 \%$ & $7.3 \%$ & $10.9 \%$ \\
\hline & $\%$ of Total & $1.0 \%$ & $1.5 \%$ & $1.2 \%$ & $2.1 \%$ & $0.7 \%$ & $2.9 \%$ & $1.4 \%$ & $10.9 \%$ \\
\hline & Count & 493 & 223 & 333 & 988 & 610 & 1675 & 1033 & 5355 \\
\hline & $\%$ within land use & $9.2 \%$ & $4.2 \%$ & $6.2 \%$ & $18.5 \%$ & $11.4 \%$ & $31.3 \%$ & $19.3 \%$ & $100.0 \%$ \\
\hline & $\%$ within cluster & $100.0 \%$ & $100.0 \%$ & $100.0 \%$ & $100.0 \%$ & $100.0 \%$ & $100.0 \%$ & $100.0 \%$ & $100.0 \%$ \\
\hline & $\%$ of Total & $9.2 \%$ & $4.2 \%$ & $6.2 \%$ & $18.5 \%$ & $11.4 \%$ & $31.3 \%$ & $19.3 \%$ & $100.0 \%$ \\
\hline
\end{tabular}

$R T Z$ residential traffic zone, $C T Z$ commercial traffic zone, $I T Z$ industrial traffic zone, $O S T Z$ open space traffic zone, NATZ natural area traffic zone, TTZ transport traffic zone

statistics show that in Eindhoven the less accessible clusters 1, 2 and 3 only occupy $9.2 \%, 4.2$ and $6.2 \%$ of total, furthermore, more than half of traffic zones are in high accessible clusters (6 and 7).

In order to estimate whether land use/land cover types and clusters are statistically independent, a Chi-square test with 30 degrees of freedom was conducted for variables in Table 3. According to the test, land use/land cover types are significantly associated with clusters. Table 3 shows that no RTZ locates in cluster 2 and cluster 3 and only a small proportion of CTZs $(1.2 \%)$ and ITZs $(8.6 \%)$ belong to these two clusters. For another less accessible cluster 1, the total of RTZs, CTZs and ITZs accounts for merely 
$6.4 \%$. On the other hand, cluster 6 and cluster 7 gather a large collection of CTZs (66.4\%), ITZs (51.4\%) and especially RTZs (85\%). The rest of RTZs, CTZs and ITZs are distributed across medium accessible clusters (4 and 5). Consequently, it can be inferred that the commercial and residential uses favor and require strong comprehensive transport support from driving, cycling and walking. The agglomeration of ITZs in cluster 4 implies that the industrial uses highly depend on motorized transport, but the needs for cycling and walking are lower than commercial and residential uses. Even cluster 3, which is less accessible for cycling and walking but medium accessible for driving, contains some ITZs.

When it comes to natural area and open space, their distributions in clusters indicate overall low requirements for transport. The demands of open space on transport are lower than industrial uses, which is implied by the fact that OSTZs in less accessible clusters (1, 2 and 3), medium accessible clusters (4 and 5) and high accessible clusters (6 and 7) account for $22.24 \%, 34.82 \%$ and $42.94 \%$ of the total, respectively, in comparison with ITZ's $10.70 \%, 37.94 \%$ and $51.36 \%$. Natural area is the second largest type among the six and all clusters have a certain number of NATZs. The great superiority of NATZs in cluster $1(59.4 \%)$, cluster $2(54.3 \%)$ and cluster $3(49.5 \%)$ corroborates the relatively discommodity of traveling to and within natural area. As for transport land, discussing the accessibility of itself serves no practical purpose. The effects of transport land on accessibility need to consider transport land type (e.g. rail or road, road rank) and network topology (e.g. circuity, complexity, connectivity). This issue is complicated and remains to be further explored.

\section{Improvements in Accessibility of S3}

As mentioned in 4.1, S1, S2, S3 (see Fig. 3c) are less accessible for driving, cycling and walking. Since S1 is an airport and S2 is a natural area, only S3, in which an industrial park locates, is able and deserves to be improved in accessibility. Urban development is a mutual adaption process between land use systems (e.g. land use/land cover pattern) and transport systems (e.g. transport infrastructure). The transport construction and land use type change are durable and very slow in highly developed economies of today, which can even have been no change in physical patterns for centuries (Simmonds et al. 2013), so that Eindhoven can adopt a quasi-equilibrium representation of development with an interval of several years or decade. It can be inferred from a normally functioning city (e.g. Eindhoven) that, through long-term evolution of land use/land cover pattern and transport infrastructure, the transport conditions of most areas can support corresponding land use/land cover types. Therefore, the transport characteristics of each land use/land cover type, which are summarized from the majority, can be the reference for assessing any adjustment to either land use systems or transport systems. In the context of this study, the adjustments all are 'conservative surgery' (Geddes 1949) without intervening the evolutionary approach of city dramatically, which is consistent with slow, incremental and local change regarding the planning of cities (Batty and Marshall 2017). The analysis of 4.2 shows that $89.30 \%$ of ITZs belongs to high accessible clusters and medium accessible clusters, whereas $10.70 \%$ of them are in less accessible clusters. Therefore, the overwhelming majority of ITZs in high and medium accessible clusters indicates that the 
mobility service level characterized by high and medium accessible clusters, especially cluster 4 and cluster 6 with the top 2 driving accessibility, is more suitable for industrial land use at this stage.

The accessibility patterns and cluster analysis enable the identification of transport incongruous area (e.g. S3) where the accessibility doesn't meet the demands of land use. To illustrate how the results from 4.1 and 4.2 can serve for urban planning and management practice, we designed two cases for improving accessibility of S3 (see Fig. 4a). As introduced in 4.1, there is no entrance \& exit on the north and south sides of S3. Case 1 is opening a new access at location A and adding a bridge to connect inside roads with urban road networks. Case 2 is an upgrade of Case 1. Case 2 involves the measures in Case 1 and opens another access at location B. Locations A and B are at both ends of an inside road, thus, Case 2 forms a path connecting inside roads with
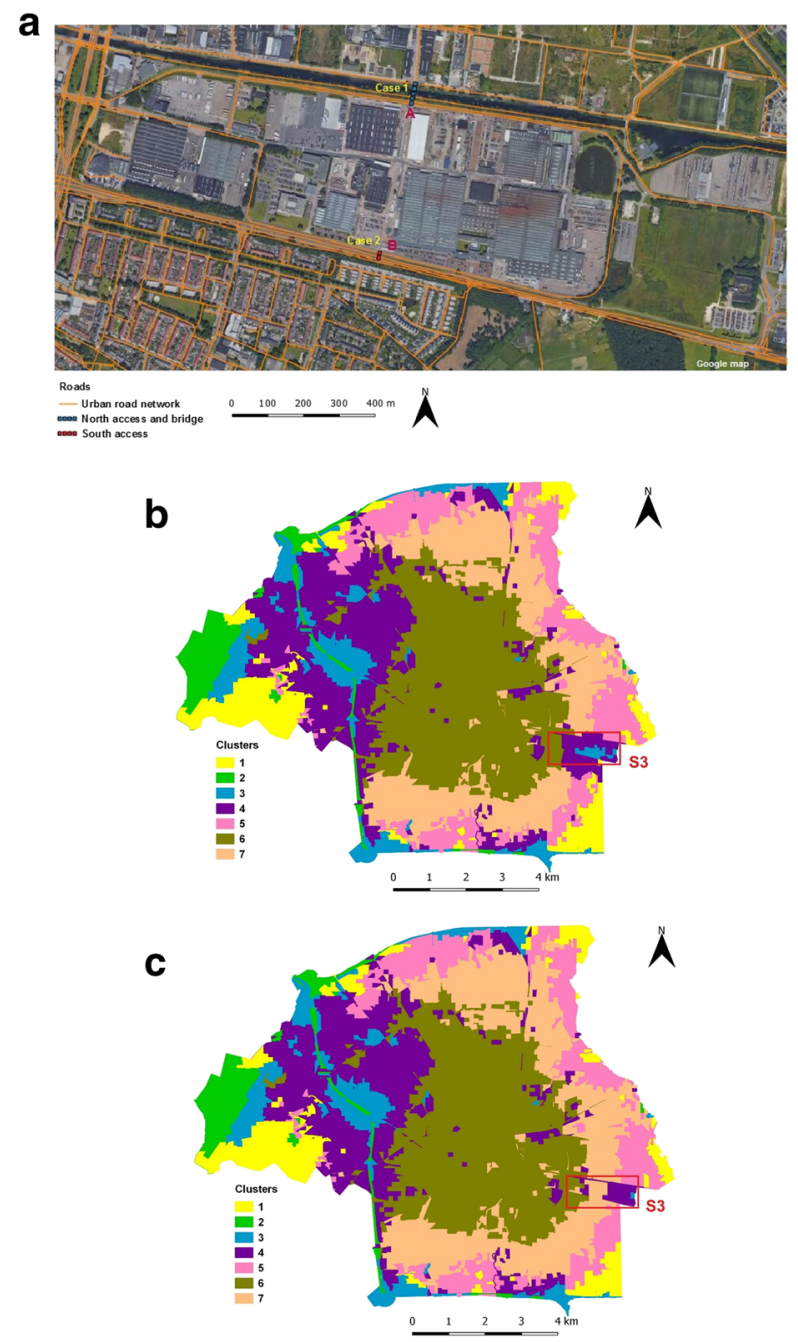

Fig. 4 Improvements in accessibility of S3: a case 1 and case 2 illustration, b subdivisions of the study area in 7 clusters under case 1 , c subdivisions of the study area in 7 clusters under case 2 
urban road networks in the north-south direction, which supplements the existing path in the west-east direction. These two cases are 'fine-tunings' of transport infrastructure under the current land use/land cover pattern. As mentioned before, infrastructure construction such as highways, major thoroughfares, railways and canals, which could provoke quantum changes in urban layout, is beyond the scope of this study.

Under Case 1 and Case 2, the driving, cycling and walking accessibility were calculated by the formulas in 3.2 for a discriminant analysis. With a priori knowledge of classifications achieved in 4.2, which is the standard for the discriminant analysis, traffic zones were re-classified into 7 clusters based on new accessibility calculations. Case 1 changes some ITZs in S3 from cluster 3 to cluster 4 (see Fig. 4b). These ITZs are accumulated in the northern part of S3, on which the access at location A and the new bridge have a direct impact. In Case 2, all traffic zones, except for one, change from cluster 3 to cluster 4 or cluster 7 (see Fig. 4c). Benefiting from accesses connecting inside roads with urban road networks in both north and south directions, the comprehensive accessibility of S3 is improved further as compared with Case 1. When land use/land cover pattern remains stable, the impact of upgrading transport infrastructure should be within limits so as not to cause large disturbance to the temporary quasi-equilibrium between transport infrastructure and land use/land cover pattern. Case 1 and Case 2 are minor modifications to existing transport systems and the main effects of them on accessibility are restricted in S3. Only a few traffic zones near urban edge but away from S3 change to higher clusters in the wake of improvements in driving convenience provided by Case 1 and Case 2.

\section{Discussion and Conclusions}

This study adopted driving, cycling and walking accessibility, which are measured by a negative exponential function of average travel time with e-number, to quantify the transport characteristics of traffic zone. The spatial units used in this study, traffic zones, were created by partitioning parcels with single land use/land cover type to investigate the relationship, if any, between land use/land cover types and accessibility. In addition to the uniqueness of land use/land cover type, the size and squarish shape of traffic zone can also handle MAUP efficiently and ensure the homogeneity of transport characteristics. The accessibility calculations were mapped as spatial accessibility patterns, all of which reveal strong positive spatial autocorrelation echoing the agglomerations of land use/land cover. The three accessibility patterns are generally various but city center is always the most accessible area. Through clustering method, driving, cycling and walking accessibility were synthesized to group traffic zones into seven clusters. According to the results of cluster analysis and contingency table analysis, it is statistically significant that land use/land cover types are associated with transport characteristics. Locations with best comprehensive accessibility for driving, cycling and walking tend to be residential and commercial uses. Industrial use prefers locations with high driving accessibility. Cycling and walking are secondary to the priority of driving accessibility for the siting of industry. Open space favors locations with high driving accessibility as well, while its demands on accessibility are comprehensively lower than industrial use. The majority of NATZs in less accessible clusters reflects the low travel generation and attraction in natural areas. 
Obviously, there is no universally applicable urban planning strategy. However, the case study of Eindhoven, a typical European city, can be valuable to other cities. This study evidences the prospects and effectiveness of cooperating accessibility measurements with clustering method in land use management and transport planning. The proposed approach can pinpoint potential locations for transport improvement or landscape alteration. To demonstrate the clustering map's utility for decision-making, we designed two cases to improve accessibility of a particular industrial area.

To achieve urban sustainability, transport infrastructure and associated mobility service should match land use and development and vice versa. The relationship between land use/land cover types and accessibility brings a new view into planning practice, e.g. transport network design and land use allocation. For transport planning, the transport systems should be upgraded to improve the mobility service of RTZs, CTZs and ITZs with insufficient accessibility, which is a shift from only emphasizing the operation of transport systems themselves to supporting land use and development. In the example of Eindhoven, the driving accessibility of ITZs which are in cluster 1, 2 and 3 requires to be improved. With regard to residential and commercial areas, the driving, cycling and walking infrastructure, any of which fails to provide adequate accessibility (e.g. transport infrastructure serve for RTZs and CTZs in medium or less accessible clusters), should be strengthened to fill the accessibility gaps. Land use and development cannot realize its full potential without the supports from transport systems. From a land use and development perspective, the main policy implications of this study are given as follows: with established transport systems, (1) residential and commercial development should be given priority to be distributed in high accessible areas (e.g. cluster 6 and 7); (2) the location of industry should satisfy its high requirements for efficient vehicular transport, e.g. cluster 4; (3) to take full advantage of excessive mobility service, the open space and natural area with comprehensive high accessibility (e.g. open space and natural area in high and medium accessible clusters) are qualified and desirable locations for landscape alterations brought on by urban development.

\section{Compliance with Ethical Standards}

Conflict of Interest The authors declare that they have no conflict of interest.

Open Access This article is distributed under the terms of the Creative Commons Attribution 4.0 International License (http://creativecommons.org/licenses/by/4.0/), which permits unrestricted use, distribution, and reproduction in any medium, provided you give appropriate credit to the original author(s) and the source, provide a link to the Creative Commons license, and indicate if changes were made.

\section{References}

Acheampong, R. A., \& Silva, E. A. (2015). Land use-transport interaction modeling: A review of the literature and future research directions. Journal of Transport and Land Use, 8(3), 11-38.

Batty, M., \& Marshall, S. (2017). Thinking organic, acting civic: The paradox of planning for cities in evolution. Landscape and Urban Planning, 166, 4-14. 
Bertolini, L., Le Clercq, F., \& Kapoen, L. (2005). Sustainable accessibility: A conceptual framework to integrate transport and land use plan-making. Two test-applications in the Netherlands and a reflection on the way forward. Transport Policy, 12(3), 207-220.

Brent, R. P. (1971). An algorithm with guaranteed convergence for finding a zero of a function. The Computer Journal, 14(4), 422-425.

Cao, K., Huang, B., Wang, S., \& Lin, H. (2012). Sustainable land use optimization using boundary-based fast genetic algorithm. Computers, Environment and Urban Systems, 36(3), 257-269.

CBS (2012). Productomschrijving Bestand Bodemgebruik. https://www.cbs.nl/nl-nl/dossier/nederlandregionaal/geografische $\% 20 \mathrm{data} /$ natuur\%20en\%20milieu/bestand-bodemgebruik.

Cervero, R., \& Duncan, M. (2003). Walking, bicycling, and urban landscapes: Evidence from the San Francisco Bay Area. American Journal of Public Health, 93(9), 1478-1483.

Chaudhuri, G., \& Clarke, K. C. (2015). On the spatiotemporal dynamics of the coupling between land use and road networks: Does political history matter? Environment and Planning. B, Planning \& Design, 42(1), 133-156.

Cliff, A. D., \& Ord, J. K. (1973). Spatial Autocorrelation. London: Pion.

Debrezion, G., Pels, E., \& Rietveld, P. (2011). The impact of rail transport on real estate prices: An empirical analysis of the Dutch housing market. Urban Studies, 48(5), 997-1015.

Duncan, M. J., Winkler, E., Sugiyama, T., Cerin, E., Leslie, E., \& Owen, N. (2010). Relationships of land use mix with walking for transport: Do land uses and geographical scale matter? Journal of Urban Health, 87(5), 782-795.

Dur, F., \& Yigitcanlar, T. (2015). Assessing land-use and transport integration via a spatial composite indexing model. International Journal of Environmental Science and Technology, 12(3), 803-816.

Duranton, G., \& Turner, M. A. (2012). Urban growth and transportation. Review of Economic Studies, 79(4), 1407-1440.

Geddes, P. (1949). Cities in evolution. London: Williams \& Norgate.

Giuliano, G., Redfearn, C., Agarwal, A., \& He, S. (2012). Network accessibility and employment centres. Urban Studies, 49(1), 77-95.

Handy, S. L., \& Niemeier, D. A. (1997). Measuring accessibility: An exploration of issues and alternatives. Environment and Planning A, 29(7), 1175-1194.

Horner, M. W., \& Schleith, D. (2012). Analyzing temporal changes in land-use-transportation relationships: A LEHD-based approach. Applied Geography, 35(1-2), 491-498.

Kenworthy, J. R., \& Laube, F. B. (1996). Automobile dependence in cities: An international comparison of urban transport and land use patterns with implications for sustainability. Environmental Impact Assessment Review, 16(4-6), 279-308.

King, D. (2011). Developing densely: Estimating the effect of subway growth on new York City land uses. Journal of Transport and Land Use, 4(2), 19-32.

Kwan, M. P., \& Weber, J. (2008). Scale and accessibility: Implications for the analysis of land use-travel interaction. Applied Geography, 28(2), 110-123.

Lambin, E. F., \& Meyfroidt, P. (2011). Global land use change, economic globalization, and the looming land scarcity. Proceedings of the National Academy of Sciences, 108(9), 3465-3472.

Lautso, K., Spiekermann, K., Wegener, M., Sheppard, I., Steadman, P., Martino, A., et al. (2004). PROPOLIS: Planning and research of policies for land use and transport for increasing urban sustainability. PROPOLIS final report. European Commission. http://www.spiekermann-wegener. de/pro/pdf/PROPOLIS_Final_Report.pdf. Accessed 15 April 2018.

Lee, C., \& Moudon, A. V. (2006). The 3Ds+ R: Quantifying land use and urban form correlates of walking. Transportation Research Part D: Transport and Environment, 11(3), 204-215.

Litman, T., \& Steele, R. (2017). Land use impacts on transport. Victoria, Canada: Victoria Transport Policy Institute.

Moran, P. A. (1948). The interpretation of statistical maps. Journal of the Royal Statistical Society: Series B: Methodological, 10(2), 243-251.

Mothorpe, C., Hanson, A., \& Schnier, K. (2013). The impact of interstate highways on land use conversion. The Annals of Regional Science, 51(3), 833-870.

Müller, K., Steinmeier, C., \& Küchler, M. (2010). Urban growth along motorways in Switzerland. Landscape and Urban Planning, 98(1), 3-12.

Murtagh, F., \& Legendre, P. (2014). Ward's hierarchical agglomerative clustering method: Which algorithms implement Ward's criterion? Journal of Classification, 31(3), 274-295.

Naumann, S., \& Kovalyov, M. Y. (2017). Pedestrian route search based on OpenStreetMap. In Intelligent Transport Systems and Travel Behaviour (pp. 87-96). Cham: Springer. 
Patarasuk, R. (2013). Road network connectivity and land-cover dynamics in Lop Buri province, Thailand. Journal of Transport Geography, 28, 111-123.

Pei, T., Sobolevsky, S., Ratti, C., Shaw, S. L., Li, T., \& Zhou, C. (2014). A new insight into land use classification based on aggregated mobile phone data. International Journal of Geographical Information Science, 28(9), 1988-2007.

Ratner, K. A., \& Goetz, A. R. (2013). The reshaping of land use and urban form in Denver through transitoriented development. Cities, 30, 31-46.

Rodríguez, D. A., Evenson, K. R., Roux, A. V. D., \& Brines, S. J. (2009). Land use, residential density, and walking: The multi-ethnic study of atherosclerosis. American Journal of Preventive Medicine, 37(5), 397-404.

Simmonds, D., Waddell, P., \& Wegener, M. (2013). Equilibrium versus dynamics in urban modelling. Environment and Planning. B, Planning \& Design, 40(6), 1051-1070.

Stanilov, K. (2003). Accessibility and land use: The case of suburban Seattle, 1960-1990. Regional Studies, 37(8), 783-794.

Tian, L., Ge, B., \& Li, Y. (2017). Impacts of state-led and bottom-up urbanization on land use change in the peri-urban areas of Shanghai: Planned growth or uncontrolled sprawl? Cities, 60, 476-486.

TomTom (2016). Traffic congestion statistics for Eindhoven based on TomTom historical database for 2016. https://www.tomtom.com/en_gb/trafficindex/city/EHV.

Vandenbulcke, G., Steenberghen, T., \& Thomas, I. (2009). Mapping accessibility in Belgium: A tool for landuse and transport planning? Journal of Transport Geography, 17(1), 39-53.

Waddell, P. (2011). Integrated land use and transportation planning and modelling: Addressing challenges in research and practice. Transport Reviews, 31(2), 209-229.

Wang, X., Lindsey, G., Schoner, J. E., \& Harrison, A. (2015). Modeling bike share station activity: Effects of nearby businesses and jobs on trips to and from stations. Journal of Urban Planning and Development, $142(1), 04015001$.

Weiss, D. J., Nelson, A., Gibson, H. S., Temperley, W., Peedell, S., Lieber, A., et al. (2018). A global map of travel time to cities to assess inequalities in accessibility in 2015. Nature, 553, 333-336.

Witten, K., Pearce, J., \& Day, P. (2011). Neighbourhood destination accessibility index: A GIS tool for measuring infrastructure support for neighbourhood physical activity. Environment and Planning A, 43(1), 205-223.

Yang, D., \& Timmermans, H. (2014). Effects of urban spatial form on individuals' footprints: Empirical study based on personal GPS panel data from Rotterdam and Eindhoven area. Procedia Environmental Sciences, 22, 169-177.

Zhang, J., Liao, F., Arentze, T., \& Timmermans, H. (2011). A multimodal transport network model for advanced traveler information systems. Procedia-Social and Behavioral Sciences, 20, 313-322. 